\title{
RECORRIDOS DE REFLEXIÓN TEÓRICA Y PRÁCTICA PARA UNA VALORACIÓN DE LAS MINORÍAS CULTURALES EN EL CONTEXTO DE LA GLOBALIZACIÓN.
}

Resumen. En este estudio se quieren presentar algunas reflexiones teóricas y practicas sobre la importancia de la valorización de las minorías culturales en el contexto de la globalización.

En la primera parte se enfrenta el tema de la sostenibilidad a partir del debate contemporáneo sobre la crítica de la racionalidad separada y a favor de una racionalidad de tipo planetario.

Se pone por lo tanto el problema del reconocimiento y de la valorización de las minorías lingüísticas y culturales cuál portadoras de importantes saberes locales que contribuyen a la construcción de la nueva forma de racionalidad.

Se pasa así a analizar la educación sostenible como una modalidad específica de hacer educación que permite el lleno desarrollo de las personas y las equipa a afrontar de manera creativa las dificultades y los desafíos de la vida.

Por fin, la atención se dirige hacia algunos proyectos internacionales que valorizan los saberes locales de las comunidades de la América Latina.

Palabras clave: Comunidad local, Educación sostenibile, Globalización, Racionalidad planetaria, Saberes locales.

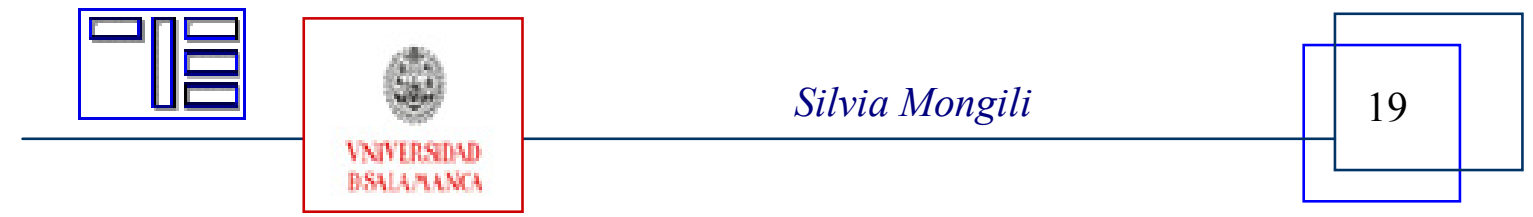


Revista Electrónica Teoría de la Educación.

Educación y Cultura en la Sociedad de la Información.

http://www.usal.es/teoriaeducacion

Vol. 9. No 2. Mayo 2008

\section{THEORETICAL AND PRACTICAL REFLECTIONS TO VALUATE CULTURAL MINORITIES IN A CONTEXT OF GLOBALIZATION.}

Abstract. The aim of this study ought to give some theoretical and practical reflections focusing on the importance of the enhancement of cultural minorities in the globalized world.

It deals with the difficult subject of sustainability starting by a reflection on the contemporary debated question on separate rationality which clashes with an ever more favourite practice of worldwide rationality.

So that the true problem seems to be the acknowledgement and the enhancement of cultural and linguistic minorities both as the keeper of important local knowledge and the builder of this new kind of rationality.

Our study goes on analyzing a kind of sustainable education which should be considered as the best way to let people expressing and developing themselves and showing them at the same time the way to live life in a creative way and to put difficulties on a brave face.

Finally the attention turns towards some international projects which set off Latin American communities' local knowledge.

Key words: Local community,. Sustainable education, Globalization, Planetary rationality, Local knowledges.

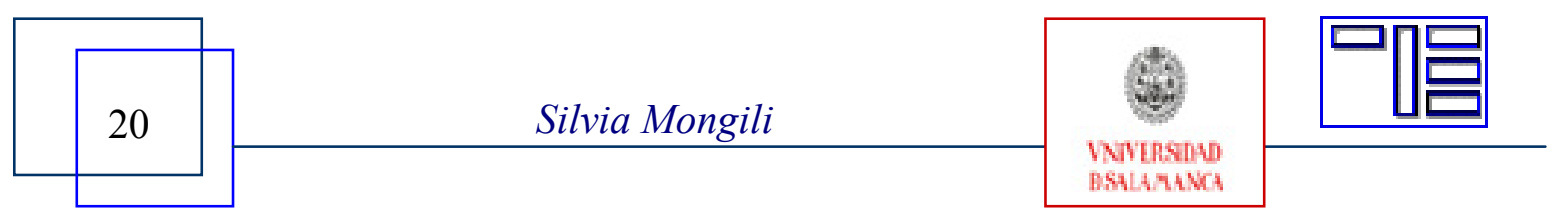




\section{PARCOURS DE RÉFLEXION THÉORIQUE ET PRATIQUE POUR UNE VALORISATION DES MINORITÉS CULTURELLES DANS LE CONTEXTE DE LA GLOBALISATION}

Sommaire. Dans cette étude sont présentées quelques réflexions théoriques et pratiques sur l'importance de la valorisation des minorités culturelles dans le contexte de la globalisation.

Dans la première partie est évoqué le sujet de la soutenibilité à partir du débat contemporain sur la critique de la rationalité séparée et au service d'une rationalité de type planétaire.

Le problème de la reconnaissance et de la valorisation des minorités linguistiques et culturelles sont donc percues comme porteuses d'importants savoirs locaux qui contribuent à la construction de la nouvelle forme de rationalité.

L'analyse porte ensuite sur l'éducation soutenable comme une modalité spécifique de faire $1^{\prime}$ éducation qui permet le développement complet des gens et les équipe à affronter de manière créatrice les difficultés et les défis de la vie.

Enfin, l'attention s'adresse vers quelques projets internationaux valorisant les savoirs locaux des communautés de l'Amérique Latine.

Mots-Clés: Communauté locale; Éducation soutenable; Globalisation; Rationalité planétaire; Savoirs locaux.

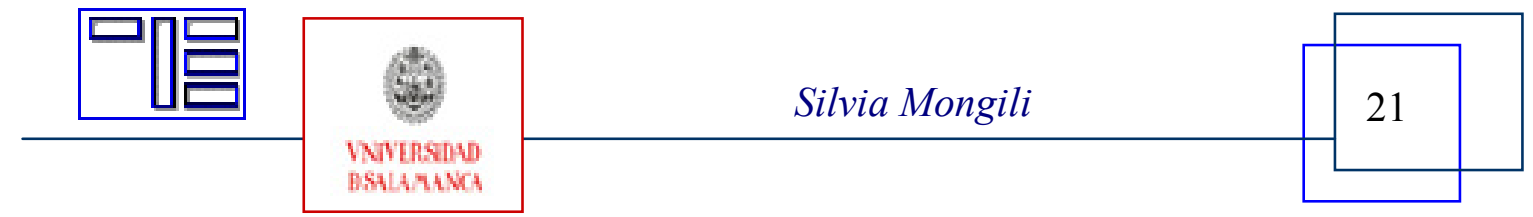




\title{
RECORRIDOS DE REFLEXIÓN TEÓRICA Y PRÁCTICA PARA UNA VALORACIÓN DE LAS MINORÍAS CULTURALES EN EL CONTEXTO DE LA GLOBALIZACIÓN.
}

\author{
Silvia Mongili \\ silvia.mongili@gmail.com \\ Universidad de Florencia (Italia)
}

\section{1.- INTRODUCCIÓN.}

La sociedad contemporánea está siendo cada vez mas atravesada por procesos de transformación ligados a la globalización: el hombre actual se encuentra inmerso en un fuerte dinamismo social, cultural y económico que conduce a la mundialización de los conocimientos e informaciones, de los modos de entender diferentes sistemas de saberes "plurales y alternativos", pertenecientes a muchos pueblos, sociedades y culturas lo que ha dado lugar tambien a la crítica a un tipo de racionalidad occidental contraponiendo a esta una forma más avanzada de racionalidad, capaz de incluir y sistematizar el vasto patrimonio cultural derivado de la experiencia del conocimiento humano. Como Orefice escribe:

" $i$ guasti della razionalità occidentale sono ormai sotto gli occhi di tutti: dai gravi danni agli equilibri ecologici ed alla vivibilità del pianeta per gli inquinamenti e per lo sfruttamento insostenibile della natura alla produzione di ricchezza e di potere all'interno delle società più industrializzate che allontana dallo sviluppo $i$ Paesi poveri, rendendoli dipendenti e subalterni all'una e all'altro; dalle nuove e diffuse malattie innescate dagli eccessi di comodità e di attivismo, come le disfunzioni cardiache, metaboliche e nervose, alle patologie sociali della massificazione e della solitudine, dell'esclusione sociale e delle nuove povertà culturali" (Orefice, 2004). ${ }^{1}$

El hecho de que en las sociedades contemporáneas la experiencia humana esté caracterizada por la complejidad e interdependencia de los fenómenos, ha generado un importante debate sobre la crítica a la racionalidad y al pensamiento ligado "a una dimensión": la disciplina científica individual por una parte, gracias a su especialización, ha sido capaz de acotar, simplificar y dividir específicamente la realidad para poder analizarla en detalles. Por otra parte, este hecho ha revelado su incapacidad de este tipo de lógica de pensamiento para articularse con otras dimensiones de la realidad misma, impidiendo muchas veces que los diferentes elementos que componen la realidad puedan dialogar entre si. En una sociedad planetaria regida por los conocimientos y los saberes, la tarea de las instituciones formativas no puede ser más la de transmitir meramente contenidos sino mas bien debe

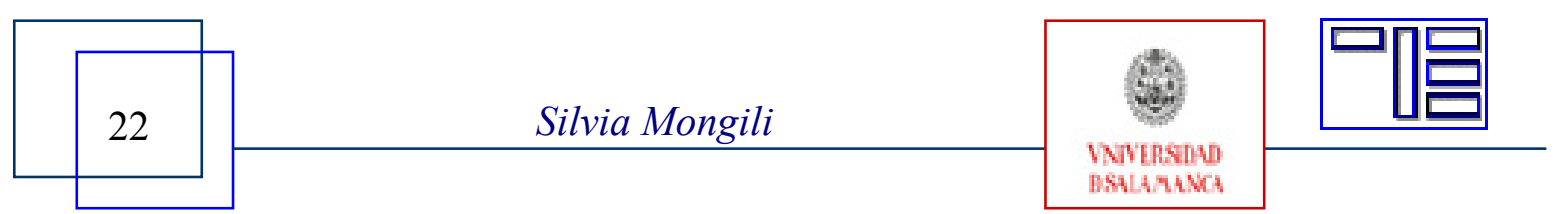


abrirse al patrimonio mundial de los conocimientos y tratar de enseñar a saberlos buscar, reconocer e implementar.

El problema histórico que se va planteando esta vinculado a la "racionalidad global", es decir un tipo de racionalidad que ya no puede validar el conocimiento en términos absolutos, y que tiene el deber de promover la construcción de un saber que corresponda e identifique a la sociedad. Se trata de pensar una educación para todos, que supere la incomunicabilidad de los saberes disciplinares -racionalidad de carácter mas occidental, a favor de un conocimiento mundial solidario que haga referencia a los conocimientos de otros grupos humanos y otras culturas que son el testimonio de que otras racionalidades son posibles para interpretar un mundo cada vez más complejo.

\section{2.- DESDE EL RACIONALISMO DISCIPLINAR A LA RACIONALIDAD PLANETARIA ${ }^{2}$}

En la sociedad actual, la educación desarrolla un papel cada vez más específico: es un motor de desarrollo social. Para edificar una verdadera sociedad democrática es importante cultivar la libertad de pensamiento en términos de producción de saberes de calidad. En la sociedad industrial los conocimientos científicos han alcanzado niveles de especialización muy elevados perdiendo de vista la complejidad y la integración de los problemas. La razón de este hecho estuvo ligada a la visión especifica y parcelada de la realidad que dieron origen a los diferentes campos de estudio y saberes disciplinares. Los conocimientos científicos, habiendo alcanzado niveles de especialización muy profundos, se mostraron capaces de solucionar problemas específicos del hombre y la naturaleza, perdiendo simultáneamente la capacidad de entrelazar los análisis y las respuestas disciplinares; y extraviando consecuentemente la visión compleja de los problemas y la integración de las respuestas.

Es evidente el posible daño causado al potencial cognoscitivo de la mente, propensa a observar desde un único punto de vista la realidad. "Il punto di arrivo è la perdita della razionalità complessiva dell'interpretazione della realtà: la critica della ragione viene spezzettata, frantumata all'interno di porzioni di realtà assunte come campo circoscritto e distinto di studio di specifici e isolati saperi disciplinari. Alla fine, le diverse critiche appaiono espressioni di altrettante ragioni che si ergono a ragione ultima della spiegazione della realtà: la disciplina assolutizza il suo punto di vista, la ragione parziale diventa ragione assoluta. La conclusione è il tradimento della libertà di pensiero" (Orefice, 2004, p. 26).

La sociedad moderna, que trató de criticar cualquier expresión del pensamiento absoluto y totalizador, ha acabado con afirmar una nueva esclavitud del pensamiento mismo, sumido en la auto-referencialidad de la cognición que impide ser libre, vale decir tiende a que los hombres queden continuamente prisioneros de si mismos dentro de una

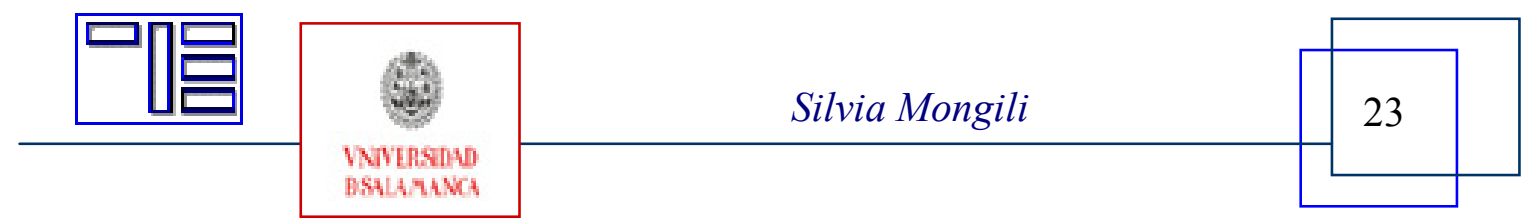


Revista Electrónica Teoría de la Educación.

Educación y Cultura en la Sociedad de la Información.

http://www.usal.es/teoriaeducacion

Vol. 9. No 2. Mayo 2008

realidad que le impide reconocer los diferentes aspectos a ella conectadas, ya sean estos puntos de vista o bien valores elementales vinculados con los procesos de aprendizaje.

La sociedad actual está experimentando el paso de una racionalidad separada hacia una racionalidad correlacionada. Por esa razón ya no es posible hablar de "especificidades separadas" sino que resulta más apropiado referir al concepto de "especificidades comunicantes". Hoy, en efecto, los grandes desafíos de la humanidad solicitan, para poder ser afrontados y analizados, el empleo de muchos conocimientos y competencias disciplinares capaces de propiciar la comunicación y operar cognoscitivamente frente a la complejidad del mundo.

La objetividad de la ciencia ya no tiene un valor absoluto sino más bien relativo y las formas de percepción es empática: el intelectual del tercer milenio tendrá que estar dotado de una mente relacional y participativa que le permita dialogar con otras personas y obrar frente a procesos complejos.

\section{3.- LA COMUNIDAD ENTRE LOCAL Y GLOBAL.}

La época actual está caracterizada por un doble fenómeno: por una parte, se evidencia el efecto creciente de la globalización que invade cada vez más ámbitos de nuestra vida; por la otra se nota como emergen cada vez más las presiones locales ejercidas por las minorías lingüísticas y culturales que solicitan mayor autonomía, descentralización y participación en el marco de un desarrollo endógeno sostenible. Ambos fenómenos pueden eventualmente derivar en autoritarismo y discriminación económica, social y cultural, si los mismos no son modulados adecuadamente.

"Il Villaggio globale può comprimere la ricchezza della diversità propria del potenziale umano e produrre uomini troppo uniformi, come altrettante marionette mosse da un oligarchico governo mondiale; il villaggio locale può fossilizzare la diversità e, annullando il potenziale umano del cambiamento, alimentare ostilità fratricide permanenti: nell'uno e nell'altro caso, compromettendo con l'abitabilità stessa del pianeta il destino dell'uomo" (Orefice, 2006, p. 4). ${ }^{3}$

Estos riesgos podrán ser abordados y reducidos gracias a un recursos importante que desde siempre forma parte de la dotación humana: se trata del poder cognoscitivo que otorga al hombre la posibilidad de entrar en relación con la naturaleza que lo circunda y con sus semejantes después de que esto fuera considerado por largas décadas como prerrogativa exclusiva de los hombres "civilizados". El reconocimiento de la propia existencia por todos los habitantes de la tierra mas allá de sus diferencias, hasta ahora no había dado lugar a que las personas pudieran ponderar y vivenciar objetivamente en sus propios conocimientos y legados culturales -merecedores de consideración y respeto.

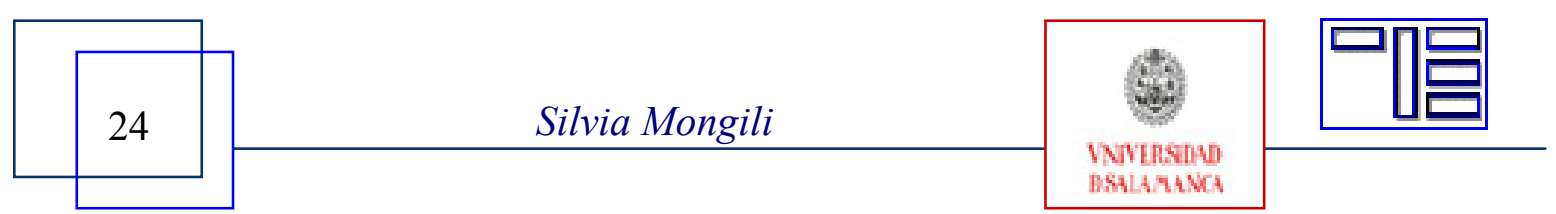


Como leemos en Berti (2005), en referencia a los efectos producidos hoy por la globalización, se asiste al redescubrimiento cada vez más fuerte de todo lo que es "local": en particular, por lo que nos concierne, parecen volver de moda las comunidades tradicionales, locales por supuesto, que se meten en contraposición a lo "global" y a favor de una continua búsqueda de espacios de vida auténtica, realmente significativos por quién los habita. Al interior de un contexto tal, la atención debe estar puesta en no caer en el error de confundir la comunidad local con un lugar privilegiado dónde se cultiva la total identidad y se excluye la diferencia y desde donde sólo se admite lo idéntico, desmedrando la apertura hacia el exterior, o sea hacia la comunidad planetaria en la que la comunidad local -valga la aclaración- se halla también inmersa.

¿Qué significado podemos atribuir hoy a lo local? Habitamos en un espacio cada vez más matizado y borroso. No existe tampoco más la comunicación espacial que hace hasta una decena de años estuvo determinada por tomar un teléfono, ha sido reemplazada por el desarrollo tecnológico y telecomunicativo virtual que ha tenido como resultado un viraje de la relación planteada entre el hombre y el territorio, entre lo próximo y lo lejano, entre lo estable y lo provisional, lo sólido y lo líquido, lo sedentario y lo nómada. Si el poder del Estado-Nación estuvo unido estrechamente a la capacidad de control sobre un determinado territorio, la característica del nuevo tiempo esta signada por la perdida del espacio fijo y la intromisión de los cánones de la versatilidad, simulteneidad e imprevisibilidad en el contexto de la globalización que constriñe todo $\mathrm{y}$ a todos. La pugna por el poder no remite mas al dominio de un territorio físico, hoy las luchas más feroces están ligadas al control de las informaciones. Estas pugnas se libran sobre incipientes "campos de batalla" y con nuevas armas como por ejemplo la informática y las nuevas tecnologías, para conseguir el control y la posesión de bienes más bien inmateriales.

En este escenario, el fortalecimiento de la comunidad local puede representar una respuesta adecuada al desafío al cual nos someten los riesgos de la sociedad contemporánea asociados a la excesiva fragmentación social y la tendencia desmedida hacia la homogeneización y simplificación de lo diverso y lo desconocido.

Hoy valorar lo local significa ponerse en contacto con el mundo entero y con las nuevas tecnologías, superando los dualismos oriente/occidente, cercano/lejano, norte/sur, regiones centrales y regiones periféricas. En esta perspectiva hace falta fortalecer la comunidad para no caer en los mismos errores del pasado y para que ella sea humanamente más sostenible. La nueva comunidad tendrá que convertirse en un lugar de encuentro y diálogo entre las diversidades, un lugar que no sea marginal y espacialmente que tenga un fuerte impacto educativo para las personas que lo habitan. La comunidad local podrá ser así la nueva vía para encontrar una mejor respuesta a las necesidades del individuo y de toda la sociedad mundial. Por este motivo, la atención a lo local sin convertirse en localismo servirá para promover comunidades basadas en la auto-observación, el espíritu crítico y la ética de la responsabilidad (Honas, 2002).

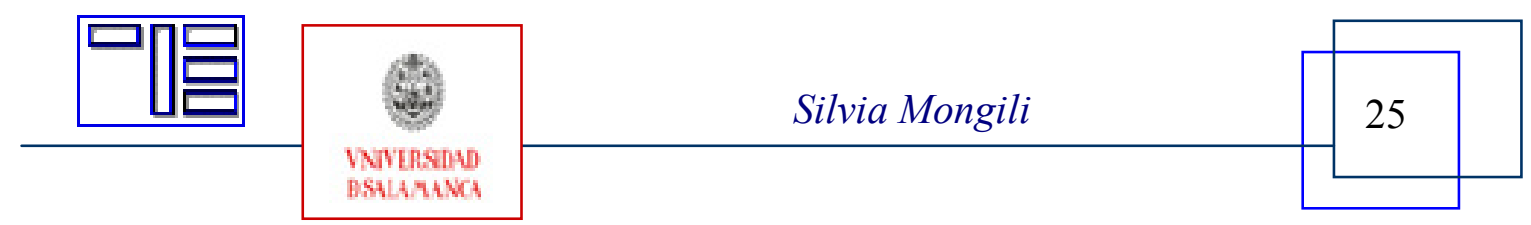


Revista Electrónica Teoría de la Educación.

Educación y Cultura en la Sociedad de la Información.

http://www.usal.es/teoriaeducacion

Vol. 9. No 2. Mayo 2008

La comunidad a la que hacemos referencia ya no puede ser, por lo tanto, aquella del pasado imbuida en la clausura de lo instituyente, la predilección por lo estático, la parcelación del mundo, pero tampoco tiene que ser una colectividad frívola, laxa y acrítica, construida sobre los principios modernos de la abstracción y el reino de lo virtual. El primer tipo de comunidad, conlleva el riesgo de quedar demasiado cerrada en ella misma y de autoexcluirse de los procesos enmendados por la economía mundial del conocimiento; la segunda por su parte expone la amenaza de ser demasiado débil al estar atravesada por uniones circunstanciales y a veces anónimas que no favorecen ni el desarrollo de la cohesión social ni la superación de los problemas y obstáculos cotidianos.

\section{4.- HACIA UNA EDUCACIÓN SOSTENIBLE.}

Dentro de este contexto se plantea el problema del reconocimiento y de la valoración de las minorías lingüísticas y culturales portadoras todas ellas de importantes conocimientos locales y que puedan contribuir a la construcción de una nueva forma de racionalidad global y relacional, la única capaz de asegurar la supervivencia armónica de la humanidad.

"In particolare, per creare una società sostenibile in armonia con gli ecosistemi locali e, insieme, al di sotto dei limiti globali, sono necessarie reti dedicate alla sostenibilità, sia a livello locale sia a livello globale [...] Una funzione delle reti locali è restituire al senso di comunità e di relazione l'importanza che aveva prima della rivoluzione industriale e che da allora è andata in gran parte perduta" (Meadows \& Randers, 2006, p. 324).

Sin lugar a dudas son muchas las acciones que habría que emprender para poder construir un mundo sostenible, pero estas acciones para ser eficaces necesitan modos de pensar completamente nuevos. Por este motivo será fundamental, entonces, posibilitar la gestación de un proceso de aprendizaje en beneficio de cada uno de los seis mil millones habitantes de la Tierra. La única forma de aprendizaje capaz de producir el cambio deseado no podrá prescindir de la consideración de todas las dimensiones del conocimiento humano, que lejos de basarse exclusivamente en las dinámicas del pensamiento se apoyan fuertemente en los sentidos y en las emociones.

"Il collasso non può essere evitato se gli uomini non imparano a considerare se stessi e gli altri come parti di una società globale integrata. In entrambi $i$ casi, c'è bisogno di solidarietà, non solo nei confronti di chi è vicino e presente, ma anche di chi è più lontano nel tempo e nello spazio. L'umanità deve imparare ad amare l'idea di lasciare alle generazioni future un pianeta vivente" (Meadows \& Randers, 2006, p. 332). ${ }^{5}$

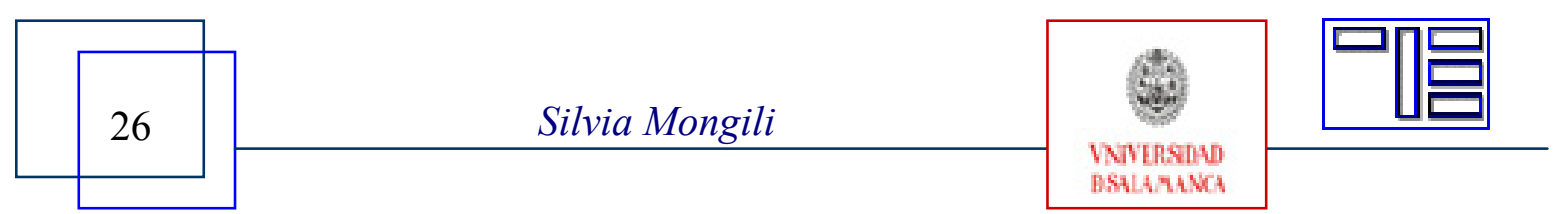


El esfuerzo pedagógico será por tanto fundamental si queremos que los habitantes de la tierra tengan la posibilidad de elegir el camino adecuado, el único que los conducirá a acercarse con amor y con respeto a sus compañeros de viaje, actual y futuros, humanos y no humanos, cercanos y lejanos. En este sentido aprender a considerar a largo plazo las consecuencias de sus acciones y de sus elecciones representa un importante desafío para los ciudadanos del mundo.

Resultan muy interesantes experiencias y conclusiones elaboradas por las acciones y postulados de la educación sostenible, que indica una modalidad de hacer educación que, además de asegurar el pleno desarrollo de las personas, prepara para afrontar de modo crítico y creativo las dificultades y los desafíos de la vida, y orienta las transformaciones tendientes a construir una sociedad mejor. Si coincidimos en que "hacer educación" significa ocuparse del desarrollo y apertura dl conocimiento en la acción educativa no se puede prescindir de las ideas y de los pensamientos que han llevado a la construcción del modo de pensar de los sujetos. En consecuencia, el educador no puede trabajar para reconducir determinados conocimientos y competencias de forma arbitraria de acuerdo a un solo patrón cultural: la dimensión pluricultural e intercultural tiene que ser considerada como parte constitutiva del desarrollo del sujeto. Expresado en otros conceptos para que la educación pueda constituirse como un instrumento fundamental de la sostenibilidad hace falta poner en practica un paradigma educativo que tenga como fundamentos la humanidad, la democracia y la ecología: tal paradigma no puede estar representado por la visión occidental de la educación, mecánica y reduccionista, orientada por la filosofía del saber como mercancía, todo lo contrario el nuevo paradigma debe avalar una ecología de la educación, que perciba el sistema educativo, su ethos, el currículo y la comunidad como un todo interrelacionado.

Una educación sostenible trasciende los límites de la educación ambiental o la educación para el desarrollo sostenible en tanto que aboga por un cambio sustancial de la tarea pedagógica. Este planteamiento educativo novedoso pretende superar el modelo transmisivo a partir de la instauración de un modelo trasformativo que reconoce las diferencias individuales y de grupo, y fortalece la comprensión de la complejidad e interdependencia del mundo contemporáneo ${ }^{61}$.

Brevemente, los valores centrales del paradigma educativo transmisivo se basan en el carácter reduccionista en como se entienden los procesos sociales, la disposición de mecanismo de selección y exclusión, la predilección por un tipo exclusivamente formal de la educación, el entendimiento del conocimiento con fuerte valor instrumental ${ }^{7}$, la exaltación de la competencia, la especialización y la integración con objetivos de adaptación, el aprendizaje transmisivo y eficaz, la estandarización, la mensurabilidad, la confianza en el sistema, la modernidad y el progreso. Estos aspectos antes enumerados están siendo afortunadamente replanteados gracias al pujante encuentro y diálogo intercultural y así también gracias a la lucha librada por la minoría étnica cultural en el mundo orientada a conquistar un mayor reconocimiento cultural y social. ${ }^{8}$

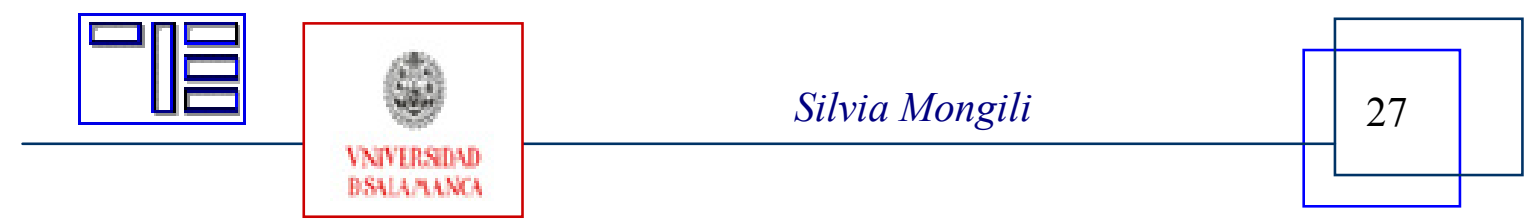


Revista Electrónica Teoría de la Educación.

Educación y Cultura en la Sociedad de la Información.

http://www.usal.es/teoriaeducacion

Vol. 9. No 2. Mayo 2008

De la otra parte, el paradigma "ecológico" aboga por un tipo de educación de carácter sostenible que valora integralmente el género humano, fomenta la participación en todas las dimensiones de la transición hacia la sostenibilidad social, económica y ambiental, apoya la inclusión de todas las personas en todos los aspectos y en todas las edades de su vida, alienta el aprendizaje a lo largo de toda la vida, la cooperación y la integración de las disciplinas. Ahora bien, si el paradigma ecológico pone un mayor énfasis en el conocimiento local, personal y aplicado y en la dimensión de comunidad, el paradigma transmisivo-reduccionista enfatiza un tipo de conocimiento descontextualizado y abstracto y, a diferencia del primer paradigma, no admite la posibilidad de articular la comunidad local y la comunidad de aprendizaje a nivel global.

\section{5.- UNA MIRADA HACIA ALGUNOS PROYECTOS INTERNACIONALES QUE VALORAN LAS COMUNIDADES LOCALES DE AMÉRICA LATINA.}

La Cátedra de Pedagogía Social de la Facultad de Ciencias de la Formación de la Universidad de los estudios de Florencia, en donde trabaja quién escribe en calidad de "doctoranda", promueve desde ya hace algunos años, importantes proyectos internacionales concernientes al desarrollo social integral, que no se limitan a hacer una descripción de la conformación geográfico-político-económica de un país determinado, sino que apuesta por la construcción de un diálogo con los actores locales.

Nuestro grupo de investigación, en efecto, paralelamente, en relevo de los intereses específicos que tienen las instituciones, las asociaciones y las comunidades locales frente al desarrollo, considera importante estudiar los nudos problemáticos intrínsecos en tales realidades para poder dar cuenta de las necesidades formativas (de su desarrollo),y la construcción de una base sólida para la formulación de planes de investigación-intervención compartida, que promueven la participación de la sociedad en todos los niveles. Consideramos el diálogo permanente como el factor básico y el vehículo de valoración de metodologías de investigación científica que facilitan en forma participativa la integración de los saberes locales propios de la población, esgrimidos -desde sus realidades y con sus potencialidades- y su articulación con lo global.

Nuestros trabajos profundizan el valor de lo material y lo inmaterial como parte del patrimonio de las comunidades, los territorios y las regiones. Por eso hablar de desarrollo endógeno implica conocer y reconocer los recursos, el capital humano, los saberes y las competencias del territorio, pensando en la inserción socio cultural de los hombres a partir de la adquisición de competencias sociales y en la responsabilidad social. Desde nuestro aporte buscamos construir un modelo de desarrollo humano, incluyente, participativo, equitativo y democrático que parta de las potencialidades del territorio y empodere a la población en articulación a lo local y lo global.

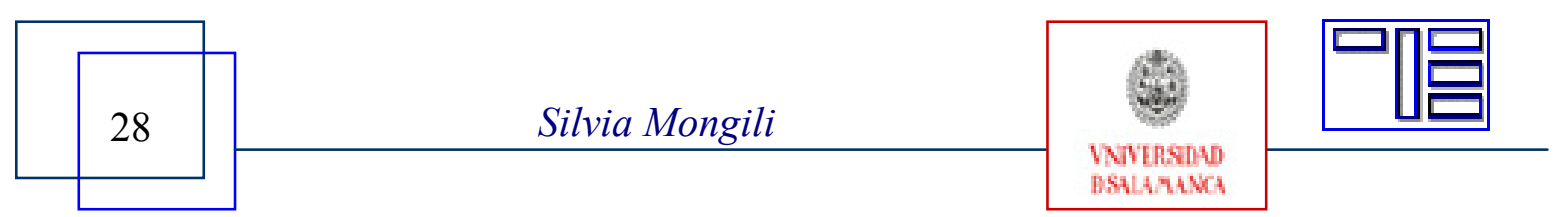


Por tal razón, creemos importante poner en común los conocimientos y los saberes a nivel interdisciplinario, desde un plano internacional, donde se unen los saberes europeos con los latinoamericanos.

5.1. Proyecto "InterlinkPlus: Potencial humano y patrimonio territorial para el desarrollo sostenible endógeno: teorías, métodos y experiencias interdisciplinarias en la relación entre saberes locales y saberes globales"

InterlinkPlus es un proyecto financiado por el Ministerio de la Universidad (MIUR), la Universidad de Florencia y otros Partners ${ }^{9}$, que esta bajo la coordinación del Prof. Paolo Orefice. Este proyecto se encuentra inserto dentro del Programa de Internacionalización del Sistema Universitario (INTERLINK), que fuera aprobado en el año 1999 por el Ministerio de la Instrucción, de la Universidad y de la Investigación (MIUR) con el objetivo de dinamizar el proceso de internacionalización de los ateneos, de promover el sistema universitario italiano en el extranjero y de incentivar programas integrados de estudio e investigación.

El Proyecto, se desarrolla en el trienio 2006-2008, y culmina con la realización de un programa de investigación que contempla la movilidad de docentes, investigadores y doctorandos (italianos y extranjeros) que disponen de una beca para la concreción de una investigación. InterlinkPlus al ser un Network de Universidades e Instituciones italianas y latinoamericanas, se propone como finalidad la elaboración y la aplicación de modelos teóricos y metodológicos contextualizados y participativos orientados a analizar y valorar el capital humano y patrimonio territorial.

Sobre el plan de las estrategias y las prácticas de investigación el Proyecto busca alcanzar un trabajo interdisciplinar, la integración entre la investigación teórica y la investigación aplicada, y el fortalecimiento de sinergias entre las actividades de investigación y las actividades de cooperación internacional.

La metodología de investigación adoptada en el proyecto utiliza tres topologías de estudio: a saber, a) la aproximación crítica-hermenéutica (modalidad de investigación profundamente introducida en los contextos operacionales de los cuales emergen las problemáticas indagadas); b) la aproximación interdisciplinaria (diferentes contribuciones disciplinarias, metodologías y contenidos, que exploran la misma compleja realidad del capital humano y del patrimonio territorial para el desarrollo endógeno sostenible); y c) la aproximación de la Investigación Acción Participativa (formación de "comunidades de autocrítica" implicadas en el proceso de conocimiento y transformación de la realidad).

El escenario en el que se mueve la investigación se apoya en los aportes de la Knowledge Society para dirimir sobre los actuales cambios globales, atendiendo a las consecuencias de la mundialización de los procesos y de los productos, ponen en evidencia los problemas de integracion preemientes en las sociedades y las culturas en

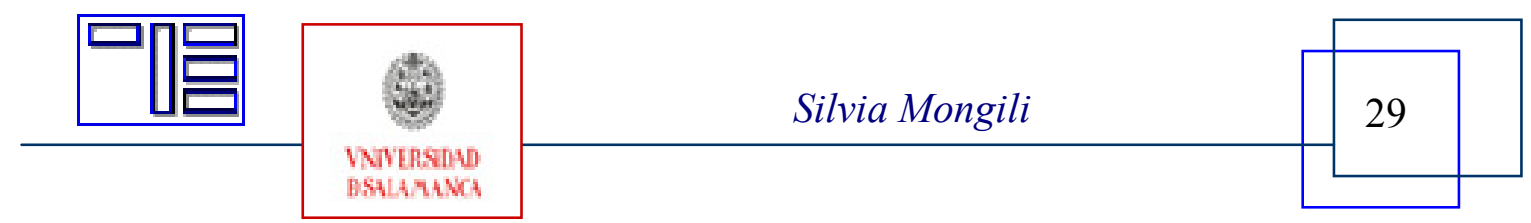


Revista Electrónica Teoría de la Educación.

Educación y Cultura en la Sociedad de la Información.

http://www.usal.es/teoriaeducacion

Vol. 9. No 2. Mayo 2008

las diversas regiones del planeta. En tal escenario, el potencial del conocimiento humano asume un significado nuevo que va más allá del valor instrumental del conocimiento que finaliza con la creación de productos materiales: al conocimiento se le ha asignado un formidable valor propulsor no solo sobre el plano cultural, sino también sobre el plano del desarrollo social y económico, sostenible e inclusivo. En tal contexto, para alcanzar los objetivos indicados, la investigación indaga sobre los siguientes ejes problemáticos y sus recíprocas relaciones en el ámbito territorial en los términos de Good Practices previo a efectuar las acciones de transferencia:

- el potencial de conocimiento expresado por los saberes colectivos indagados en cuanto dotación del capital humano de las sociedades y culturas de las áreas territoriales en examen: esta dotación expresa el capital inmaterial o producción ideal aprendido a través de la educación informal, no formal y formal;

- el patrimonio arqueológico y territorial expresión de los saberes de las sociedades y culturas de las áreas en estudio: esta dotación expresa el capital material o producción de material de cuya utilización vienen alimentadas las expresiones y las estructuras de aprendizaje colectivo que confluyen en las diversas formas de educación y determinan los procesos de socialización y culturalización;

- el desarrollo sostenible endógeno considerado como dirección dinámica y estructural del cambio con la finalidad de construir una Polis de autogobierno que abogue por la inclusión y del bienestar de los ciudadanos, a través de procesos de Institutional Building que culminan con la edificación de Local Societies de Knowledge/Lifelong Learning. Ahora bien, desde la teoría y estrategia ligada al del cambio endógeno se exploran actualmente los procesos de cambio global que por un lado, vehiculizan la reproducción de las sociedades y culturas tecnológicamente más avanzadas, con los relativos saberes globales producidos (comprendidos aquellos científicos) y por otro lado, subrayan la importancia de los saberes colectivos y de los patrimonios territoriales locales de las sociedades y culturas emergentes, pero condicionadas por el desarrollo dependiente.

La Unidad de Investigación del Departamento de Ciencias de la Educación de la Universidad de Florencia, coordinado por el Prof. Orefice, participa en InterlinkPlus a través del Proyecto de desarrollo educativo local e integrado en Mugello (Comunidad Montana, Cooperativa El Forteto), se fundamenta en la teoría de la construcción de los saberes locales, del sentir y del pensar, y de igual modo adopta la metodología de la Participatory Action Research como base de referencia para la construcción de la Sociedad local del conocimiento (Cité Educative) a través de la valorización del patrimonio material e inmaterial del distrito interurbano de la comunidad de Montana. Las Acciones del Proyecto local insertadas en InterlinkPlus abarcan áreas del trabajo escolar y extraescolar y el campo de la educación de los adultos, en muchos niveles: las estrategias y las redes del sistema educativo local articulado con las prácticas de formación y de trabajo en colaboración con los operadores locales e integrando

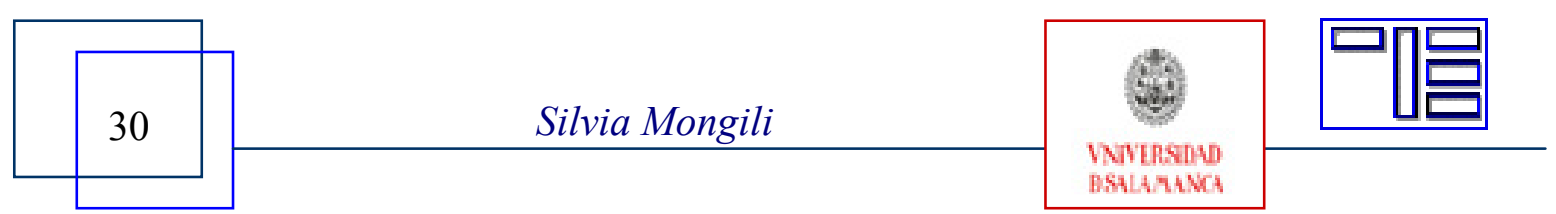


metodológica y técnicamente la educación formal, no formal e informal en el ámbito de formación de jóvenes y adultos.

\subsection{Proyecto "U-Maya: Firenze e la Toscana per l'università Maya"}

El proyecto U-maya es un proyecto de cooperación destinado al sostén a la constitución de una Universidad Maya y esta ligado desde sus génesis con temas relacionados a la globalización y la defensa de los derechos de las culturas marginales. El proyecto fue gestado a partir del Foro Social de Florencia celebrado en noviembre de 2002, en aquella ocasión Rigoberta Menchù (premio Nobel de la Paz) fue invitada por el Rector de la Universidad de Florencia a discutir sobre problemas de la globalización, las dinámicas culturales y los riesgos que pueden causar la falta de reconocimiento de los derechos de las culturas marginales o minoritarias. En este evento la atención se centró fuertemente en la cultura maya, que derivo al finalizar en una solicitud explicita de colaboración científica y profesional para poder edificar una Universidad Maya en Guatemala.

El proyecto ha sido financiado por la Región Toscana, la Provincia de Florencia, el Ayuntamiento de Florencia y el Ayuntamiento de Pontassieve. La Universidad de Florencia se ha comprometido en la efectivización de este proyecto bajo la coordinación científica del Prof. Paolo Orefice y la implicación de un grupo de docentes, investigadores y doctorandos vinculados al Departamento de Ciencias de la Educación y de los Procesos Culturales y Formativos, Departamento de Economía Agraria y de los Recursos Territoriales, Departamento de Ingeniería Agraria Forestal (DIAF) y del CSIAF (Centro Servicios Informáticos del Ateneo florentino). Del mismo modo dos ONG's han sido integradas en la realización del proyecto, las mismas son Mani Tese, encargada de la coordinación administrativa, y Action Aid Internacional, que posee una amplia presencia institucional en Guatemala, y garantiza por lo mismo el monitoreo de las acciones. En Guatemala se han incorporado como cooperantes el Consejo Nacional de Educación Maya (CNEM), la Fundación Rigoberta Menchù Tum (FRMT), la Academia de Lengua Maya Guatemala (ALMG) y el Instituto Superior de Desarrollo Integrado Local (ESEDIR).

El Proyecto tiene como finalidad la realización de una Universidad Maya en Guatemala según un modelo de Universidad comunitaria que busca preservar la cultura e instaurar un espacio abierto para la reflexión e investigación sobre las problemáticas interculturales y la globalización. Este proyecto representa una importante posibilidad de intervención sobre las diferentes problemáticas que nacen a partir de la posición de sometimiento que padecen los pueblos indígenas en Guatemala. Entre las finalidades del proyecto figura la posibilidad de garantizar el reconocimiento de los derechos específicos de las poblaciones indígenas para la superación de la situación de discriminación y exclusión, favorecer el acceso a la educación superior a los jóvenes indígenas y de sustentar la formación de una adecuada clase dirigente maya, y promover

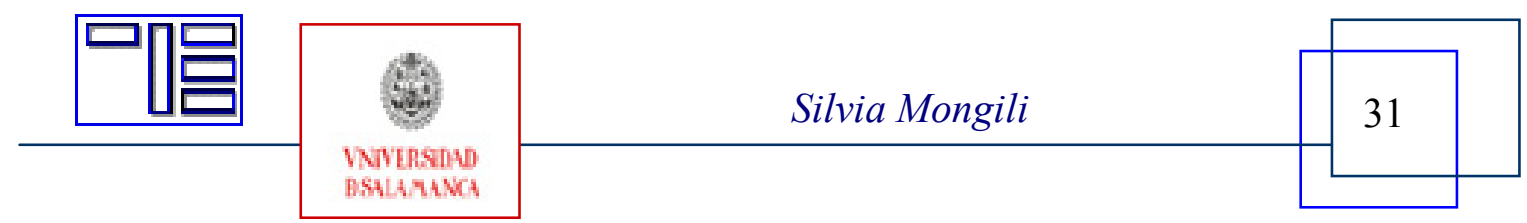


el desarrollo socio-económico y cultural de las comunidades indígenas fomentando la participación de las poblaciones en la vida social, económica y cultural del país.

La tarea hasta ahora desarrollada por los actores implicados en el proyecto ha posibilitado un interesante trabajo de investigación y desarrollo de los recursos humanos, y la elaboración de propuestas para conservar el patrimonio de saberes que están en peligro de desaparición.

En el proyecto adquiere también una relevancia particular el sentido que puede asumir la formación superior dentro de un Estado todavía caracterizado por discriminaciones respecto a la población local indígena, que representa la mayoría de la población guatemalteca. La idea que mueve este y otros proyectos análogos en Guatemala, es encontrar formas y modalidades funcionales para no homogeneizar todas las culturas presentes, vale decir reconociendo que cada pueblo posee una identidad y tiene derechos legítimos de determinación sobre su patrimonio cultural dentro de un estado nación.

Resulta por tanto de fundamental importancia apoyar en un país como Guatemala la conservación de la diversidad cultural, una diversidad que se manifiesta en su multiplicidad de rostros y expresiones culturales, variados y enriquecedores.

\section{6.- BIBLIOGRAFÍA.}

ABBAGNANO, N. (2006). Dizionario di filosofia, cur. G. Foriero. Torino: UTET.

BAUMAN, Z. (2004). Voglia di comunità. Bari-Roma: Laterza.

BAUMAN, Z. (2005). Dentro la globalizzazione. Le conseguenze sulle persone. BariRoma: Laterza.

BAUMAN, Z. (2005). Fiducia e paura nella città, Milano: Bruno Mondadori Editore.

BERTI, F. (2005). Per una sociologia della comunità. Milano: Franco Angeli.

BOCCHI, G. \& CERUTI, M. (2004). Educazione e globalizzazione. Milano: Raffaello Cortina Editore.

BOLOGNA, G. (2005). Manuale della sostenibilità. Idee, concetti, nuove discipline capaci di futuro. Milan: Edizioni Ambiente.

CAPRA, F. (1990). II punto di svolta. Scienza, società e cultura emergente. Milano: Feltrinelli.

CAPRA, F. (2005). La rete della vita. Milano: BUR Scienza.

CARRINO, L. (2005). Perle e pirati. Trento: Erickson.

DEL GOBBO, G. (2007). Dall'educazione all'ambiente. Pisa: Del Cerro.

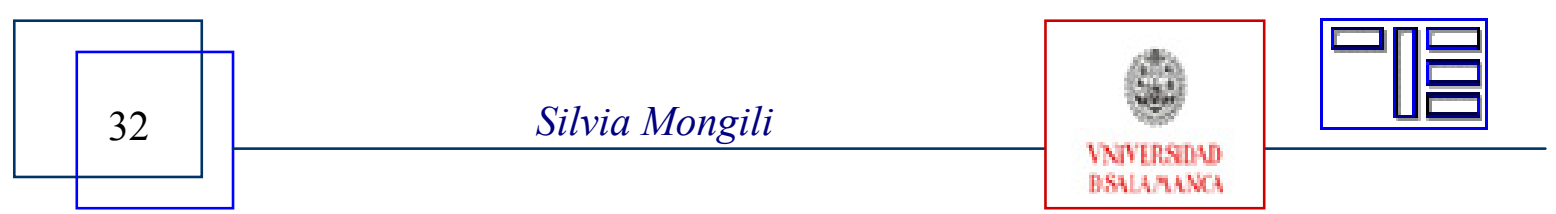


JONAS, H. (2002). Il principio responsabilità. Un'etica per la civiltà tecnologica. Torino: Einaudi.

LA CAMERA, F. (2003). Sviluppo sostenibile: origini, teoria e pratica. Roma: Editori Riuniti.

MALAVASI, P. (a cura di) (2003). Per abitare la Terra, un'educazione sostenibile. Milano: I.S.U. Università Cattolica.

MALAVASI, P. (a cura di) (2005). Pedagogia dell'ambiente. Milano: I.S.U. Università Cattolica.

MCMICHAEL, P. (2006). Ascesa e declino dello sviluppo. Milano: Franco Angeli.

MEADOWS, D. y D., MEADOWS, L., RANDERS, J. \& BEHRENS III, W. (1972). I limiti dello sviluppo. Milano: Oscar Mondadori.

MEADOWS, D. y D. \& RANDERS, J. (2006). I nuovi limiti dello sviluppo. Milano: Oscar Mondadori.

MORIN, E. (1993). Introduzione al pensiero complesso. Milano: Sperling \& Kupfer Editori.

MORIN, E. (2000). La testa ben fatta. Milano: Cortina.

MORTARI, L. (1994). Abitare con saggezza la terra. Forme costitutive dell'educazione ecologica. Milano: Franco Angeli.

MORTARI, L. (2001). Per una pedagogia ecologica. Milano: La Nuova Italia.

OREFICE, P. (2001). I domini conoscitivi. Roma: Carocci.

OREFICE, P. (2006). La Ricerca Azione Partecipativa. Teoria e pratiche. Vol. I e II. Napoli: Liguori.

OREFICE, P. (2006). Pedagogia. Roma: Editori Riuniti.

OREFICE, P. \& SARACINO, V. (a cura di) (2004). Nuove questioni di pedagogia sociale. Milano: Franco Angeli.

RIFKIN, J. (2000). L'era dell'accesso. Milano: Mondadori.

SACHS, W. (a cura di) (2004). Dizionario dello sviluppo. Torino: EGA Editore.

SEN, A. (2000). Lo sviluppo è libertà. Perché non c'è crescita senza democrazia. Milano: Arnoldo Mondadori Editore.

SEN, A. (2004). La democrazia degli altri. Perché la libertà non è un'invenzione dell'Occidente. Milano: Arnoldo Mondatori Editore.

The World Commission on Environment and Development (1987). Our Common Future, Oxford University Press.

TIZZI, E. \& MARCHETTINI, N. (1999). Che cos'è lo sviluppo sostenibile? Le basi scientifiche della sostenibilità e i guasti del pensiero unico. Roma: Donzelli.

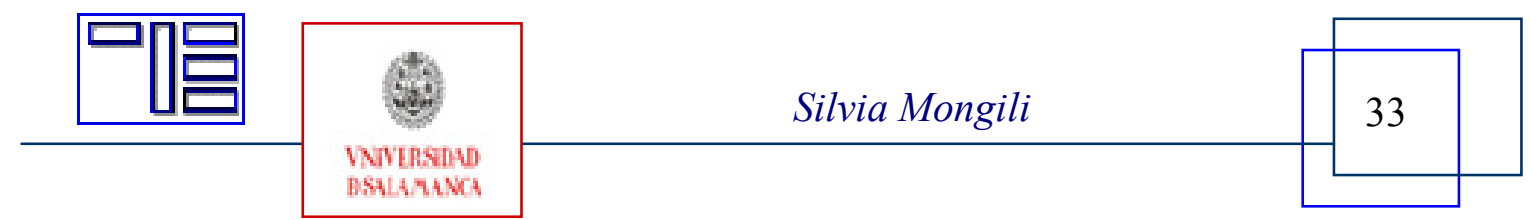


Revista Electrónica Teoría de la Educación.

Educación y Cultura en la Sociedad de la Información.

http://www.usal.es/teoriaeducacion

Vol. 9. No 2. Mayo 2008 UNDP (1990-2005). Lo Sviluppo Umano. Rapporti Annuali. Torino: Rosenberg \&
Sellier.

\section{Notas}

${ }^{1}$ Cfr. Orefice P., Società, educazione e conoscenza: dal razionalismo disciplinare alla razionalità planetaria, Orefice P., Saracino V. (a cura di), Nuove questioni di pedagogia sociale, Franco Angeli, Milano, 2004, p. 24. Traducción: "las averías de la racionalidad occidental son ya bajo los ojos de todos: desde los graves daños a los equilibrios ecológicos y a la habitabilidad del planeta por las poluciones y por la explotación insostenible de la naturaleza hasta la producción de riqueza y poder dentro de las sociedades más industrializadas que aleja del desarrollo los Países pobres, devolviéndolos dependientes y subalternos a la una y al otro; desde las nuevas y difusas enfermedades cebadas por los excesos de comodidad y activismo como las disfunciones cardíacas, metabólicas y nerviosas, hasta las patologías sociales de la masificación y la soledad, de la exclusión social y de las nuevas pobrezas culturales".

${ }^{2}$ Orefice, P.: Società, educazione e conoscenza: dal razionalismo disciplinare alla razionalità planetaria, Orefice, P. y Saracino, V. (a cura di) (2004): Nuove questioni di pedagogia sociale, Milano, Franco Angeli, pp. 15-47. Traducción:"El punto de llegada es la pérdida de la racionalidad total de la interpretación de la realidad: la crítica de la razón es troceada, triturada dentro de porciones de realidad asumidas como sector circunscrito y distinguido de estudio de específicos y aislados saberes disciplinares. Al fin, las muchas críticas aparecen expresiones de mismas razones que se yerguen a razón última de la explicación de la realidad: la disciplina impone su punto de vista, la razón parcial se convierte en razón absoluta. La conclusión es la traición de la libertad de pensamiento."

${ }^{3}$ Cfr. Orefice, P. (2006): La Ricerca Azione Partecipativa. Teoria e pratiche, Volume secondo, Napoli, Liguori, p. 4. Traducción:"La Aldea global puede comprimir la riqueza de la diversidad propia del potencial humano y producir hombres demasiado uniformes, como marionetas movidas por un oligárquico gobierno mundial; la aldea local puede fosilizar la diversidad y, anulando el potencial humano del cambio, alimentar hostilidades fratricidas permanentes: en el uno y en el otro caso, comprometiendo con la habitabilidad misma del planeta la suerte del hombre".

${ }^{4}$ Cfr. Meadows, D. y D.; Randers J. (2006): I nuovi limiti dello sviluppo, Milano, Oscar Mondadori, p. 324. Traducción: "En particular, para crear una sociedad sostenible en armonía con los ecosistemas locales y también debajo de los límites globales, son necesarias redes dedicadas a la sostenibilidad, sea a nivel local sea a nivel global [...] Una función de las redes locales es devolver al sentido de comunidad y relación la importancia que tuvo antes de la revolución industrial y que desde entonces ha ido en gran parte perdida".

${ }^{5}$ Ib., p. 332. Traducción: "El colapso no puede ser evitado si los hombres no aprenden a considerar sí mismos y los otros como partes de una sociedad global integrada. En ambos casos, hay necesidad de solidaridad, no sólo respecto a quien es cercano y presente, pero también a quien es más lejano en el tiempo y en el espacio. La humanidad tiene que aprender a amar la idea de dejar a las generaciones futuras un planeta viviente".

${ }^{6}$ Esta nueva concepción de la educación está atada estrechamente al paradigma de ciencia contemporánea. Es importante, a este propósito, subrayar como el concepto occidental de conocimiento científico es cambiado en el tiempo, pasando del paradigma propio de la ciencia declarativa, basado sobre el dogma, al paradigma de la ciencia demostrativa, basado sobre la razón, y al paradigma de la ciencia autocorrectiva basada sobre el principio de autocorrección de la ciencia (Popper). A. este propósito se haga referencia a

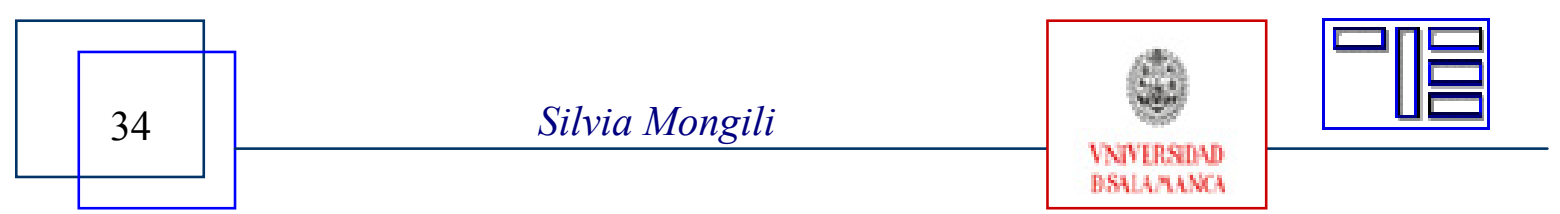


Abbagnano, N. (2006): Dizionario di filosofia, a cura de Giovanni Fornero, Torino, UTET; Popper K. (1981): Logica della scoperta scientifica, trad. M. trinchero, Torino, Einaudi; Popper, K. (1985): Congetture e confutazioni, cur. G. Pancaldi, Bologna, Il Mulino; Kuhn, T. (1978): La struttura delle rivoluzioni scientifiche, trad. A. Cargo, Torino, Einaudi; Feyerabend, P. (1985): Contro il metodo, trad. L. Sosio, Milano, Feltrinelli.

${ }^{7}$ Un ejemplo es dado por el encuentro con la cultura maya, que tiene entre sus caracteres distintivos el paradigma holístico que considera el ser humano parte de un cosmos mucho más amplio. Los seres humanos, dentro de una tal visión del mundo, están en estrecha relación con los elementos del universo y con los elementos de la naturaleza. Por éste es importante la integración de la persona con la naturaleza y con el cosmos.

${ }^{8}$ Es decir orientada a un objetivo práctico, a un producto. En el mundo occidental amenaza de llevar a una forma de educación mercantilizada y privatizada. Se haga referencia, a este propósito, a Rifkin, J. (2000): L'era dell'accesso, Milano, Mondadori.

${ }^{9}$ Partners italianos: FIRENZE (Departamento de Ciencias de la Educación, Departamento de Urbanística y Planificación del Territorio, Departamento de Historia del Arquitectura y de las Ciudades, Facultad de Letras y Filosofía), PADOVA (Departamento de Ciencias de la Educación), NAPOLI "Federico II" (Departamento de Ciencias Relacionales), NAPOLI "Parthenope" (Facultad de Ciencias Motorias), SIENA (Departamento de Economía Política), REGION TOSCANA (Dirección general de la Presidencia, sector de las actividades internacionales), UNOPS EDINFODEC ITALIA. Partner extranjeros: BRAZIL "Universidad del Estado de Bahía" (Departamento de Educación), GUATEMALA "Universidad San Carlos" (Ciudad del Guatemala - Departamento de Estudios de Postgrado), NICARAGUA "Universidad Nacional Autónoma Nicaragua (UNAN) de León" (Rectorado), NICARAGUA "Universidad Americana UAM" (Facultad de Arquitectura), CUBA “Instituto Superior Politécnico J.A. Echeverría” de La Habana (Facultad de Arquitectura).

Para citar este artículo puede utilizar la siguiente referencia:

MONGILI, Silvia (2008). Recorridos de reflexión teórica y práctica para una valoración de las minorías culturales en el contexto de la globalización. En APARICIO, Pablo (Coord.) Desde la diversidad hacia la desigualdad: ¿destino inexorable de la globalización? [monográfico en línea]. Revista Electrónica Teoría de la Educación: Educación y Cultura en la Sociedad de la Información. Vol. 9, $\mathrm{n}^{\mathrm{o}}$ 2. Universidad de Salamanca. [Fecha de consulta: dd/mm/aaaa]. $<$ http://www.usal.es/ teoriaeducacion/rev_numero_09_02/n9_02_mongili.pdf > ISSN 1138-9737

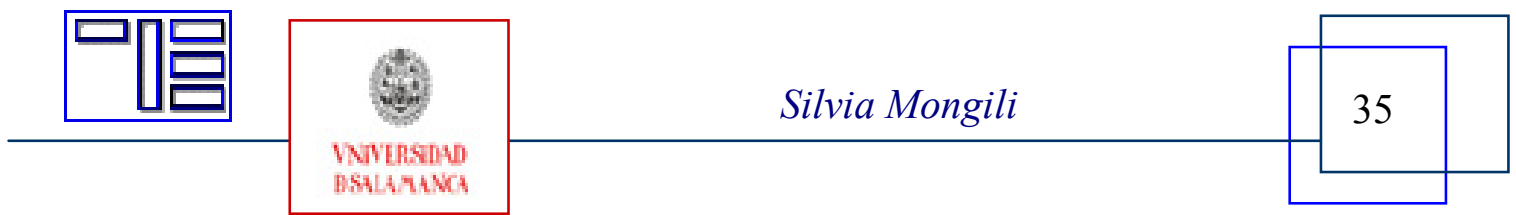

